\title{
Was können wir aus Krisen lernen? Reflexion der Verflech- tungen der Corona-Pandemie und der Klimakrise
}

\author{
*ulrike.ohl@geo.uni-augsburg.de, Institut für Geographie, Universität Augsburg \\ ** fabian.pettig@uni-graz.at, Institut für Geographie und Raumforschung, Universität Graz
}

eingereicht am: 15.06.2021, akzeptiert am: 02.08.2021

\begin{abstract}
Sowohl die Corona-Pandemie als auch die Klimakrise verdeutlichen: Die menschliche Gesundheit ist untrennbar mit der planetaren Gesundheit verbunden. Den hierin liegenden Herausforderungen und Fragen nach der Notwendigkeit einer sozial-ökologischen Transformation widmet sich die virtuelle Podiumsdiskussion des ,Thementags Klimawandel' auf der geographischen Fachtagung \#GeoWoche2021. Dort diskutieren die Geograph*innen Elke Hertig, Thomas Loster, Luisa Neubauer und Patrick Sakdapolrak die Fragestellung „Was können wir aus der Corona-Pandemie für den Umgang mit der Klimakrise lernen?” In diesem Beitrag werden Möglichkeiten aufgezeigt, diese Diskussion (als Liveübertragung am 07.10.2021 oder im Nachhinein als Videoaufzeichnung) in den Geographie- und GW-Unterricht einzubinden. Das Unterrichtsmaterial bietet Schüler*innen im Sinne politischer Bildung Gelegenheiten, um Gemeinsamkeiten, Verflechtungen und Herausforderungen der Krisen fachlich zu erschließen, eigene Standpunkte zur Fragestellung der Diskussion zu etablieren und das Expert*innengespräch kritisch zu reflektieren.
\end{abstract}

Keywords: Corona, Klimakrise, Planetare Gesundheit, Sozial-ökologische Transformation, Politische Bildung

\section{What can we learn from crises? A reflection on the interconnectivity of the COVID-19 pandemic and the climate crisis}

Both the COVID-19 pandemic and the climate crisis illustrate: Human health is inextricably linked to planetary health. This connectedness refers to challenges of our time and to the need for a social-ecological transformation, to which the virtual panel discussion of the 'theme day climate change' at the geographic symposium \#GeoWoche2021 is dedicated. There, geographers Elke Hertig, Thomas Loster, Luisa Neubauer and Patrick Sakdapolrak discuss the question „What can we learn from the pandemic for coping with the climate crisis?". This paper presents opportunities to incorporate this discussion (as a live broadcast on 07.10 .2021 or afterwards as a video recording) into geography and GW lessons. The teaching material offers students - in the sense of political education - the opportunity to learn about the commonalities, interconnections and challenges of the crises, to establish their own points of view on the question of the discussion and to critically reflect on the expert discussion.

Keywords: corona, climate crisis, planetary health, social-ecological transformation, political education

\section{Corona-Pandemie, Klimakrise und planetare Gesundheit}

"Both crises are testing our resilience and humanity in extreme ways. [...]. Yet it remains to be seen whether societies and governments around the world are willing to take up the challenge that climate change poses as vigorously as they are addressing coronavirus. In fact, it is a matter of intergenerational social justice." (Vinke et al. 2020: 6)
Das vorliegende Unterrichtsbeispiel widmet sich dem Zusammenhang der Corona-Pandemie und der Klimakrise, diskutiert deren Verflechtungen, Gemeinsamkeiten und Herausforderungen, und reflektiert unterschiedliche Standpunkte und mögliche zukunftsfähige Strategien anhand der Einbindung einer virtuellen Podiumsdiskussion in den Unterricht.

Die Klimakrise wird zunehmend auch als Gesundheitskrise diskutiert, da „die menschliche Gesundheit, Tiergesundheit und eine gesunde Umwelt eng mitein- 
ander verbunden" sind (Liedtke et al. 2020: 79). Der Lancet-Bericht ${ }^{1}$ aus dem Jahr 2020 verdeutlicht die umfassenden Verknüpfungen von Gesundheit und Klimawandel (vgl. Watts et al. 2021): Lebensräume von Mensch und Tier rücken aufgrund menschlicher Einflüsse enger zusammen und steigern die Wahrscheinlichkeit der Übertragung tierischer Erreger auf den Menschen; die globale Ernährungssicherheit ist angesichts zunehmender Dürreperioden und aufgrund von Bränden gefährdet; Hitzewellen, die für bestimmte Menschen als Gesundheitsrisiko einzustufen sind, treten häufiger und stärker auf. Diese Zusammenhänge verdeutlichen, dass Strategien des Klimaschutzes unmittelbar auch der Gesundheitsförderung dienen.

Bestimmte Regionen und Bevölkerungsgruppen zeigen sich dabei weniger resilient, d.h. widerstands- und anpassungsfähig, gegenüber Pandemie und Klimakrise. Diese Tatsache kann nicht losgelöst von globalen Macht- und Herrschaftsverhältnissen gedacht werden, wie durch das Konzept der „imperialen Lebensweise" verdeutlicht wird (vgl. Brand \& Wissen 2017). Dieses Konzept beschreibt, dass „das bessere Leben, das [...] bestimmten Menschen an spezifischen Orten [...] [möglich ist], die Untergrabung der Lebensbedingungen anderer Menschen an anderen Orten voraussetzt" (ebd.: 61). In anderen Worten: Der hohe Lebensstandard eines gewissen Teils der Weltbevölkerung wird zu Lasten von Mensch und Natur andernorts überhaupt erst ermöglicht und fortwährend reproduziert. Auf die Corona-Pandemie bezogen werden etwa angesichts unterschiedlicher Pandemieverläufe (bspw. mit Blick auf den Zugang zu Impfstoffen im Globalen Norden gegenüber dem Globalen Süden) auch Fragen nach globaler Verantwortung und Gerechtigkeit laut.

Die Corona-Pandemie hat uns deutlich vor Augen geführt, dass ein business as usual keine zukunftsfähige Option ist und es einen tiefgreifenden gesellschaftlichen Wandel auf unterschiedlichen Ebenen braucht. So kann die Pandemie auch als Zäsur im Denken und damit als Chance verstanden werden, soziale, institutionelle, wirtschaftliche, technologische und kulturelle Systeme mit Blick auf den Erhalt planetarer Gesundheit und globaler Gerechtigkeit, i. S. einer sozial-ökologischen Transformation, zu prüfen, neuzudenken und umzugestalten (vgl. Paula \& Mar 2020). Dies wirft Fragen nach der Verantwortung des*der Einzelnen und den Grenzen individueller Verantwortung

\footnotetext{
Der Lancet Countdown ist ein jährlich erscheinender Bericht zu den gesundheitlichen Folgen des Klimawandels, an dessen Erstellung 120 Vertreter*innen von 35 akademischen Institutionen und EU-Agenturen beteiligt sind. Eine anschauliche Übersicht zum Bericht ist unter https://www.lancetcountdown.org/2020report/ einsehbar.
}

angesichts global verflochtener Nicht-Nachhaltigkeit auf, die nach einem grundlegenden systemischem Wandel verlangt.

Vor diesen Hintergründen widmet sich die zentrale Podiumsdiskussion auf der \#GeoWoche2021 der Frage, was wir aus der Corona-Pandemie für den Umgang mit der Klimakrise lernen können. Die virtuelle Fachtagung für Geograph*innen aus unterschiedlichen Bereichen (Wissenschaft, Praxis, Schulbildung) ist das Alternativformat für den pandemiebedingt ausgefallenen Deutschen Kongress für Geographie 2021. Auf dem Podium diskutieren Elke Hertig, Professorin für regionalen Klimawandel und Gesundheit (Universität Augsburg), Thomas Loster, Dipl. Geograph (ehemals Rückversicherung Munich Re), Luisa Neubauer, Klimaaktivistin (Fridays for Future), und Patrick Sakdapolrak, Professor für Bevölkerungsgeographie und Demographie (Universität Wien). Die Vertreter*innen bilden damit unterschiedliche Zugänge der Geographie wie auch unterschiedliche Gruppen ab (Wissenschaftler*innen, Praktiker*innen, Student*innen, Aktivist*innen).

Das vorliegende Unterrichtsbeispiel verfolgt das Ziel, die Podiumsdiskussion in den Geographie- und GW-Unterricht zu integrieren. Die Schüler*innen haben die Möglichkeit, die Diskussion am 07.10.2021 live zu verfolgen und eigene Wortmeldungen einzubringen, oder mit einer Aufzeichnung der Diskussion zu arbeiten. Die Unterrichtsmaterialien bieten den Schüler*innen die Gelegenheit, sich fachlich auf die Podiumsdiskussion vorzubereiten, diese aktiv zu verfolgen und im Nachgang zu reflektieren. Der Idee einer emanzipatorischen Nachhaltigkeitsbildung folgend, wird das Anliegen verfolgt, Schüler*innen eine kritisch-reflexive Auseinandersetzung mit Expert*innenmeinungen zu ermöglichen und hierüber eigene, begründete Standpunkte zum Zusammenhang von Klima- und Covid-19-Krise sowie den daraus resultierenden individuellen und gesellschaftlichen Herausforderungen $\mathrm{zu}$ formulieren. Im Sinne kritischer politischer Bildung zielt das Beispiel darauf ab, über eigene Wortmeldungen an der Expert*innendiskussion zu partizipieren und Expert*innenwissen kritisch zu prüfen. Damit geht es im Beispiel zentral um die Teilhabe an der gesellschaftlichen Aushandlung einer sozial-ökologischen Transformation.

\section{Unterrichtsbeispiel}

Zur Einbindung der Podiumsdiskussion in den Geographie- und GW-Unterricht werden drei Bausteine für je eine Unterrichtsstunde formuliert, die unterschiedlich eingesetzt werden können (siehe Tab. 1). 
Tab. 1: Unterrichtsverlauf zur Einbindung der Podiumsdiskussion

\begin{tabular}{clll} 
Baustein & Schwerpunkt & Inhalte & Material \\
\hline 1 & Vorbereitung & $\begin{array}{l}\text { Problemzusammenhänge und inhaltliche Grundlagen erar- } \\
\text { beiten; zu Kernthesen der Diskutant*innen positionieren }\end{array}$ & M1, M2, AB1 (M3-M5) \\
2 & $\begin{array}{l}\text { Podiumsdiskussion } \\
\text { (Live/Aufzeichnung, } \\
\text { ggf. Hausübung }\end{array}$ & $\begin{array}{l}\text { an der Podiumsdiskussion teilnehmen; Argumentationen } \\
\text { nachvollziehen und reflektieren; anschließend erneut zu } \\
\text { Kernthesen positionieren }\end{array}$ & AB2 (M6) \\
Diskussion und & $\begin{array}{l}\text { Expert*innenpositionen kritisch reflektieren, eigene The- } \\
\text { Reflexion }\end{array}$ & $\begin{array}{l}\text { sen formulieren und die beiden Krisen in einen größeren } \\
\text { Bedeutungszusammenhang einordnen }\end{array}$ & M7 \\
\hline
\end{tabular}

Sofern eine Teilnahme an der Live-Übertragung realisiert wird, bietet das Unterrichtsbeispiel die Möglichkeit, gemeinsam mit den Schüler*innen Wortbeiträge zu erarbeiten und diese in die Live-Diskussion einzubringen. Die Podiumsdiskussion wird nach der Live-Übertragung am 07.10.2021 als Aufzeichnung zur Verfügung gestellt. ${ }^{2}$ Es besteht somit auch die Möglichkeit, die Diskussion als Hausübung anzusehen bzw. Ausschnitte gemeinsam im Unterricht anzusehen und die Reflexion direkt anzuschließen. Das Material zur Umsetzung eignet sich für beide Varianten.

\section{Baustein 1: Vorbereitung der Podiumsdiskussion}

Im Einstieg beschreiben die Schüler*innen eine Grafik zum Rückgang der Treibhausgasemissionen seit 1990 (angegeben in $\mathrm{CO}_{2}$-Äquivalenten) (M1). Anschließend formulieren sie unter Bezug auf die Überschrift („Deutschland hat das Klimaziel für 2020 erreicht - dank Corona") mögliche Gründe, die sich für diese Entwicklung anführen lassen. Auf der Hand liegt vor allem die Reduktion des Individualverkehrs (sowohl PKW als auch Flugzeug) im Zuge der Corona-Bestimmungen, bspw. durch die Zunahme an Home-Office-Konzepten.

Anschließend bietet sich eine Diskussion darüber an, ob diese Art von Verzicht auch nach der Pandemie eine mögliche, realistische und aus Sicht der Schüler*innen wünschenswerte Strategie für den Klimaschutz ist. An passender Stelle kann die Lehrkraft als Impuls ein Zitat des Untergeneralsekretärs der Abteilung für Wirtschaft und Soziales der Vereinten Nationen, Liu Zhenim, in den Blick rücken. Darin wird die CoronaPandemie als eine Art Training für den Umgang mit der weitaus umfassenderen Klimakrise charakterisiert, was zu weiteren Diskussionsbeiträgen inspirieren kann (M2). Anhand des Zitats kann unter Bezug auf die Formulierung ,transitions needed to build a healthier, more resilient and more sustainable world“

\footnotetext{
2 Die Aufzeichnung wird über den Youtube Kanal der \#GeoWoche2021 abrufbar sein: https://www.youtube.com/channel/UCucvV1JKCLsaoU9qjZ8kBWw
}

auch das Konzept der (sozial-ökologischen oder Großen) „Transformation“" ${ }^{3}$ eingeführt werden, welches sicherlich auch in der Podiumsdiskussion erwähnt wird.

Das Zitat wirft zugleich auf einer allgemeineren Ebene die Frage nach den Gemeinsamkeiten beider Krisen auf, auf die die Lehrkraft nun die Aufmerksamkeit lenken und zugleich über die stattfindende Podiumsdiskussion informieren kann: „Die meisten Menschen sind vermutlich nicht bereit, ihr Leben lang den gleichen Verzicht zu üben wie während der ,Lockdowns“. Aber vielleicht können wir doch einiges aus den Erfahrungen mit der Pandemie lernen, was uns für den Umgang mit der Klimakrise helfen kann. Genau darum geht es in einer Podiumsdiskussion, die Expert*innen bei der \#GeoWoche2021 führen bzw. führten. Doch welche Gemeinsamkeiten haben die beiden Krisen denn überhaupt? Nennt gemeinsame Merkmale der beiden Krisen, die euch einfallen. Erklärt, inwiefern diese Merkmale tatsächlich auf beide Krisen zutreffen." Folgende Gemeinsamkeiten können die Schüler*innen möglicherweise aufgrund ihres Vorwissens nennen und erläutern: beide Krisen haben eine globale Dimension - alle Teile der Erde sind (unterschiedlich stark) davon betroffen; deshalb ist zum Umgang mit beiden Krisen eine internationale Zusammenarbeit wichtig; beide Krisen sind verursacht oder verstärkt durch den nicht-nachhaltigen Umgang der Menschen mit der Natur (Klimakrise: anthropogener Treibhauseffekt, Coronakrise: z. B. Verringerung der Lebensräume von Wildtieren, dadurch steigende Gefahr, sich bei infizierten Wildtieren anzustecken).

Auf dieser Grundlage können nun die Leitfragen der Unterrichtssequenz formuliert werden: In welchem Zusammenhang stehen beide Krisen? Was kann man aus der Corona-Pandemie für den Umgang mit der Klimakrise lernen?

\footnotetext{
3 Das Konzept der ,Großen Transformation' beschreibt einen epochalen Umbruchprozess auf unterschiedlichen Ebenen (u.a. Ökologie, Technologie, Ökonomie ...) zu Beginn des 21. Jahrhunderts. Mit dem Zusatz ,sozial-ökologisch`wird die Richtung dieses Wandels skizziert - in Richtung einer gerechten und nachhaltigen Zukunft.
} 
In der anschließenden Unterrichtsphase vertiefen die Schüler*innen ihre fachliche Auseinandersetzung mit den Gemeinsamkeiten beider Krisen sowie mit möglicherweise übertragbaren Lösungsstrategien anhand von Informationstexten (M3, vgl. Aufgabe 1 auf dem Arbeitsblatt). Diese greifen Inhalte auf, die den aktuellen wissenschaftlichen, gesellschaftlichen und politischen Diskurs zur Frage, was wir aus der Pandemie für den Umgang mit der Klimakrise lernen können, prägen. Da dieser Diskurs wiederum sehr aktuell und dynamisch ist, stellt das Material zwangsläufig eine Auswahl denkbarer Argumentationen dar. Ggf. bietet sich eine zusätzliche eigene Recherche an, um die jeweils aktuellsten Diskussionsstränge zu integrieren.

Auf dieser Grundlage setzen sich die Schüler*innen anschließend mit Kernthesen, die die Teilnehmer*innen der Podiumsdiskussion im Vorfeld der Veranstaltung formuliert haben (M4), auseinander. Entsprechend ihrer individuellen Sichtweisen, ihres Vorwissens und unter Bezug auf die Inhalte von M3 beurteilen und begründen sie anhand eines Meinungsstrahls (M5), wie stark sie den unterschiedlichen Thesen zustimmen (vgl. Aufgaben 2 und 3 auf dem Arbeitsblatt). Im Plenum können die individuellen Zuordnungen verglichen und diskutiert werden. Dies soll die Schüler*innen bei ihrer persönlichen Meinungsbildung unterstützen und sie dazu motivieren, an der Live-Übertragung der Podiumsdiskussion teilzunehmen bzw. deren Aufzeichnung anzusehen. $\mathrm{Zu}$ diesem Zweck formulieren sie nun (individuell, in Gruppen oder gemeinsam im Plenum) nach ihrem persönlichen Interesse eigene Fragen und Kommentare, die sie gerne in die Diskussion einbringen möchten bzw. würden (vgl. Aufgabe 4 auf dem Arbeitsblatt).

\section{Baustein 2: Podiumsdiskussion}

Die Schüler*innen sehen sich die Podiumsdiskussion (als Liveübertragung oder als Aufzeichnung) an, bringen sich bei einer Teilnahme an der Liveübertragung mit ihren vorbereiteten Wortbeiträgen ein und identifizieren Aussagen der Diskussionsteilnehmer*innen, die hinsichtlich ihrer vorbereiteten eigenen Fragen und Kommentare relevant sind (vgl. Aufgabe 5 auf dem Arbeitsblatt). Zur Vorbereitung der gemeinsamen Reflexion im Plenum (Baustein 3) notieren sie Argumente aus der Diskussion, die sie als besonders oder gar nicht überzeugend einschätzen (vgl. Aufgabe 6 auf dem Arbeitsblatt).

Im Anschluss an die Podiumsdiskussion nehmen sie, wieder anhand eines Meinungsstrahls (M6), eine erneute Beurteilung der im Vorfeld erarbeiteten Kernthesen der Diskussionsteilnehmer*innen vor.
Sie erläutern und begründen im Sinne einer aktiven Meinungsbildung unter Bezug auf die Inhalte der Podiumsdiskussion, ob und weshalb sie (keine) Veränderungen hinsichtlich ihrer Zuordnungen vorgenommen haben (vgl. Aufgabe 7 auf dem Arbeitsblatt).

\section{Baustein 3: Reflexion der Podiumsdiskussion}

\section{Schritt 1}

In der Klasse wird gesammelt, bei welchen Thesen sich die Beurteilungen der Schüler*innen besonders deutlich verändert haben (vgl. vorbereitende Aufgabe 7 auf dem Arbeitsblatt). Dazu kann z. B. für jede These abgefragt werden, bei wie vielen Schüler*innen sich besonders deutliche Veränderungen in Richtung einer noch stärkeren oder geringeren Zustimmung ergeben haben. So wird deutlich, welche Thesen interessant für die Reflexion sind. Letztere bezieht sich auf die Frage, aus welchen Gründen sich die Einschätzungen verändert haben. Dabei kann sowohl auf der inhaltlichen Ebene argumentiert werden (z. B. Benennung besonders überzeugender oder wenig überzeugender Argumente der Diskussionsteilnehmer*innen, Beleuchtung neu erkannter inhaltlicher Zusammenhänge oder aufgelöster Missverständnisse) als auch auf die Überzeugungskraft einzelner Diskutant*innen fokussiert werden (z. B. auch unter Berücksichtigung von Sympathie oder Körpersprache).

\section{Schritt 2}

Auf Grundlage dieser intensiven Auseinandersetzung mit dem Thema der Podiumsdiskussion können die Schüler*innen nun ihre persönliche Antwort auf die zweite Leitfrage der Unterrichtseinheit wie auch eine eigene Kernthese formulieren. Der Arbeitsauftrag lautet: Wir haben uns nun den Gemeinsamkeiten und Verflechtungen der beiden Krisen und möglicherweise übertragbaren Umgangsweisen gewidmet. Verfasse vor diesem Hintergrund nun

a) deine persönliche Antwort auf die zweite Leitfrage der Unterrichtssequenz: „Was kann man aus dem Umgang mit der Corona-Pandemie für den Umgang mit der Klimakrise lernen?” (5 Sätze).

b) eine eigene, präzise Kernthese, um deinen Standpunkt griffig zu verdeutlichen. Schreibe diese auf ein großes Blatt, das du im Klassenzimmer aufhängst.

Jede*r Schüler*in erhält nun vier Klebepunkte (alternativ können die Punkte auch aufgemalt werden), die frei auf die Thesen der Mitschüler*innen verteilt werden können und Zustimmung ausdrücken. Abschließend kann ein Austausch über die so deutlich gewordenen Einschätzungen stattfinden. 


\section{Schritt 3}

Den Abschluss der Unterrichtssequenz stellt eine Diskussion zum Zusammenhang zwischen Coronaund Klimakrise anhand einer in den sozialen Medien häufig geteilten Karikatur dar (M7). Anders als zuvor werden dabei nicht die Gemeinsamkeiten oder Lösungsstrategien aufgrund der Erfahrungen mit einer Krise für den Umgang mit einer anderen Krise diskutiert, sondern die weitergehenden Verflechtungen beider Krisen in den Blick genommen. Es soll verdeutlicht werden, wie die Klimakrise unmittelbar mit der planetaren und damit menschlichen Gesundheit zusammenhängt. Die Schüler*innen beschreiben die Karikatur, bevor diese interpretiert wird und Kernaussagen gemeinsam bestimmt werden. Drei zentrale Aspekte lassen sich herausarbeiten: Kurzsichtigkeit bei der Krisenbewältigung (verdeutlicht durch die Sprechblase), Kausalität der Krisen (verdeutlicht durch die Abfolge der Wellen), Gefahrenpotenziale der Krisen (verdeutlicht durch die unterschiedliche Wellenhöhe). Abschließend nehmen die Schüler*innen kritisch zu den zentralen Aussagen der Karikatur Stellung und ordnen diese - auch vor dem Hintergrund der Podiumsdiskussion - ein.

\section{Literatur}

Brand, U. \& M. Wissen (2017): Imperiale Lebensweise. Zur Ausbeutung von Mensch und Natur im globalen Kapitalismus. oekom, München.
Künzel, V. \& L. Schäfer (2020): Fünf Lehren aus der Coronakrise. Katastrophenmanagement und gesellschaftliche Widerstandsfähigkeit. In: oekom e.V. - Verein für ökologische Kommunikation (Hrsg.): Die Coronakrise. Stimulanz für die Große Transformation? oekom, München. S. 63-68.

Lenton, T., H. Held, E. Kriegler, J. Hall, W. Lucht, S. Rahmstorf \& H. J. Schellnhuber (2008): Tipping elements in the Earth's climate system. In: Proceedings of the National Academy of Sciences 105(6). S. 1786-1793. DOI: https://doi.org/10.1073/pnas.0705414105

Liedtke, T. P., C. Hornberg \& T. Mac Call (2020): One Health. In: oekom e.V. - Verein für ökologische Kommunikation (Hrsg.): Die Coronakrise. Stimulanz für die Große Transformation? oekom, München. S. 76-81.

Paula, N. de \& K. A. Mar (2020): Ist das Coronavirus "gut" für den Klimawandel? Die Frage geht am Thema vorbei. Potsdam. https://www.iass-potsdam.de/de/ blog/2020/03/coronavirus-klimawandel (05.06.2021)

PIK (Potsdam-Institut für Klimafolgenforschung) (2019): Kipppunkte im Klimasystem. Eine kurze Übersicht. Potsdam. http://www.pik-potsdam.de/-stefan/Publications/Kipppunkte\%20im\%20Klimasystem \%20-\%20 Update\%202019.pdf (07.06.2021)

Vinke, K., S. Gabrysch, E. Paoletti, J. Rockström \& H. J. Schellnhuber (2020): Corona and the climate: a comparison of two emergencies. In: Global Sustainability 3(e25). S. 1-7. DOI: https://doi.org/10.1017/sus.2020.20

Watts, N., M. Amann, N. Arnell, S. Ayeb-Karlsson, A. Costello et al. (2021): The 2020 report of The Lancet Countdown on health and climate change: responding to converging crises. In: The Lancet 397(10269). S. 129-170. DOI: https://doi.org/10.1016/S0140-6736(20)32290-X 


\section{Materialien für den Unterrichtseinstieg}

M1 | Corona als Katalysator des Klimaschutzes?

\section{$\operatorname{taz} 8$}

\section{Deutschland hat das Klimaziel für 2020 erreicht - dank C rona}

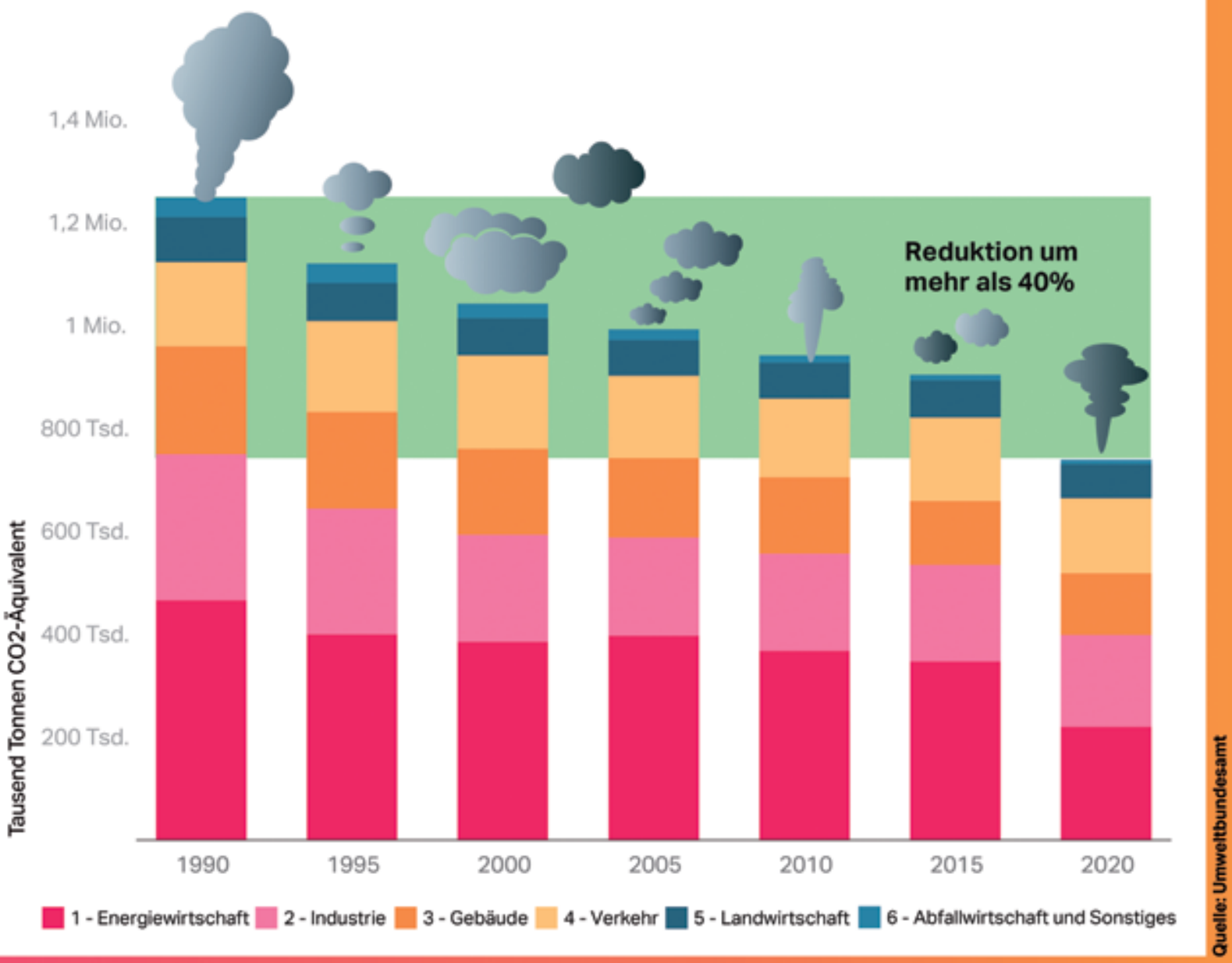

(taz; 28.03.2021 via Instagram)

\section{M2 | Der Umgang mit der Pandemie als Übung für den Umgang mit der Klimakrise?}

Im Vorwort des Sustainable Development Goals Report 2020 schreibt Liu Zhenim, Untergeneralsekretär der Abteilung für Wirtschaft und Soziales der Vereinten Nationen:

“Our collective response to the pandemic can serve as a 'warm-up' for our preparedness in preventing an even larger crisis - that is, global climate change, whose effects are already becoming all too familiar. Governments and businesses should heed the lessons learned from this wake-up call to formulate the kinds of transitions needed to build a healthier, more resilient and more sustainable world.” (UN 2020:3) 


\title{
Arbeitsblatt 1: Vorbereitung der Podiumsdiskussion
}

\author{
Was können wir aus der Corona-Pandemie für den Umgang mit der Klimakrise lernen?
}

\section{Aufgaben:}

1. Ihr habt euch überlegt, was die Corona-Pandemie und die Klimakrise gemeinsam haben. Lest nun die Informationstexte (M3) und vergleicht eure eigenen Ideen mit den Informationen aus M3. Markiert dazu mit einer Farbe die dargestellten Gemeinsamkeiten und solche Argumente, die auch in euren Ideen enthalten waren, und mit einer anderen Farbe die wichtigsten für euch neuen Informationen.

2. Die Expert*innen, die an der Podiumsdiskussion teilnehmen werden, haben schon vor der Diskussion Kernthesen zur Frage formuliert, was wir aus der Corona-Pandemie für den Umgang mit der Klimakrise lernen können (M4). Lest die acht Thesen und ordnet sie dann auf dem Meinungsstrahl (M5) zu. Hinweis zum Meinungsstrahl: Überlegt, welcher These ihr wie stark zustimmt. Wenn ihr einer These sehr stark zustimmt, schreibt ihr das entsprechende Kürzel (z. B. „EH 2“, „TL 1") sehr weit links auf den Meinungsstrahl. Je weniger ihr einer These zustimmt, desto weiter rechts schreibt ihr das entsprechende Kürzel auf den Meinungsstrabl.

3. Wählt nun zwei Thesen aus, die ihr besonders interessant findet und die ihr an unterschiedlichen Stellen auf dem Meinungsstrahl verortet habt. Begründet für diese beiden Thesen eure Zuordnungen jeweils schriftlich in zwei bis drei Sätzen. Bezieht dabei die Informationen aus M3 und euer eigenes Vorwissen mit ein.

4. Welche Fragen oder Kommentare zum Thema der Podiumsdiskussion kommen euch nun selbst in den Sinn? Formuliert eine oder mehrere Wortmeldungen (Fragen, Kommentare), mit denen ihr euch in die Podiumsdiskussion einbringen könnt.

\section{M3 | Corona- und Klimakrise: Fünf Gemeinsamkeiten}

\section{Bei beiden Krisen spielen sog. „Kipppunkte“ eine Rolle}

Beide Krisen machen rasches Handeln erforderlich, weil in beiden Fällen Kipppunkte erreicht werden können. Ein Kipppunkt (engl. tipping point) ist ein kritischer Grenzwert, an dem eine kleine zusätzliche Störung zu einer qualitativen Veränderung im System führen kann (vgl. Lenton et al. 2008; PIK 2019). Es ist also der Moment, in dem sich eine bisherige Entwicklung durch bestimmte Ereignisse und Rückkopplungen stark verändert oder beschleunigt; dabei können unumkehrbare Prozesse in Gang gesetzt werden.

Wenn die globalen Temperaturen weiter ansteigen, so können zum Beispiel die Permafrostböden, also die dauerhaft gefrorenen Böden der Polarregionen und einiger Gletschergebiete, tauen. Diese enthalten, davon gehen Wissenschaftler*innen aus, „wahrscheinlich 50\% des gesamten im Boden gespeicherten Kohlenstoffs weltweit" (PIK 2019). Dieser Kohlenstoff stammt aus den Überresten von Tieren und Pflanzen, die seit der letzten Eiszeit dort eingelagert wurden. Als Folge des Tauens wird der in den Permafrostböden eingeschlossene Kohlenstoff von Mikroorganismen abgebaut und freigesetzt. Es gelangen also große Mengen von Kohlenstoffdioxid und Methan in die Atmosphäre, wodurch die Erderwärmung deutlich verstärkt wird. Als Beispiel für einen möglichen Kipppunkt bei der Corona-Pandemie wird eine die Anzahl der Krankenhausbetten übersteigende Anzahl von Erkrankten genannt (vgl. Künzel \& Schäfer 2020: 64). Wie es sich in der dramatischen Situation in Indien im Frühling 2021 zeigte, können durch die Überlastung des Gesundheitssystems unkontrollierbare Folgen entstehen.

\section{Beide Krisen verdeutlichen und verschärfen Ungleichheiten in und zwischen Staaten}

Staaten und Regionen, die über eine größere Finanzkraft verfügen, können negative gesellschaftliche und wirtschaftliche Auswirkungen von Krisen abfedern: „Länder mit funktionierenden sozialen Sicherungssystemen und finanziellen Stoßdämpfern wie Notfallbudgets und Eventualkrediten sind besser in der Lage, ihre Wirtschaft und verwundbare Gruppen zu schützen“ (Künzel \& Schäfer 2020: 66). In der Coronapandemie stellten die reichen Staaten z.B. den Wirtschaftsbetrieben umfassende Finanzhilfen zur Verfügung oder schafften es in kürzerem Zeitraum, dass größere Teile ihrer Bevölkerung geimpft wurden. 
Ärmere Menschen, die am Rand der Gesellschaft leben und die (wie häufig in den Ländern des Globalen Südens) keine staatlichen Hilfen erhalten, sind besonders krisengefährdet, da sie geringe oder keine finanziellen Polster oder Zugänge zu Risikoversicherungen haben. Sie sind durch die Krisen existenziell bedroht (z. B. aufgrund eines Jobverlusts durch die Coronapandemie oder wenn sie wegen steigender Trockenheit nicht mehr die gleichen Ernteerträge erzielen können). Maßnahmen müssen auf Hilfen abzielen, die die Resilienz (also die Widerstandskraft gegen Krisen) in bestimmten Regionen und unter bestimmten Bevölkerungsgruppen stärken. Um die Ungleichheiten zwischen Staaten nicht weiter zu verschärfen, sind im Sinne globaler Gerechtigkeit bei beiden globalen Krisen gemeinsame, internationale Strategien anstelle von nationalen Alleingängen erforderlich.

\section{Wissenschaftliche Erkenntnisse bilden das Fundament für einen zielführenden Umgang mit beiden Krisen}

Nur die Wissenschaft mit ihren Methoden der Erkenntnisgewinnung kann die Ursachen und Wirkungen der beiden Krisen systematisch erfassen und die jeweiligen Risiken abschätzen. Auf dieser Grundlage können Strategien des Umgangs entwickelt werden. Für beide Krisen gilt dabei, dass sich auch Wissenschaftler*innen nicht immer völlig einig sind und dass auf dem Weg zu wissenschaftlichen Erkenntnissen immer auch gewisse Unsicherheiten auftreten können. Schließlich gehört zur wissenschaftlichen Erkenntnisgewinnung immer auch dazu, dass mit dem Aufkommen neuer Verfahren bzw. Informationen, bestimmte Erkenntnisse unter Umständen auch neu bewertet und ggf. überarbeitet werden müssen. Dennoch zeigte sich in der Coronakrise: „Länder, in denen die wissenschaftlichen Erkenntnisse ernst genommen und früh zur Handlungsgrundlage gemacht wurden, konnten die Coronakrise effektiver eindämmen" (Künzel \& Schäfer 2020: 64). Mit Blick auf den Klimawandel sind die wissenschaftlichen Erkenntnisse sehr umfassend und inzwischen gut abgesichert. In den Sachstandsberichten des Weltklimarats IPCC (Intergovernmental Panel on Climate Change) sind diese dargestellt.

\section{Bei beiden Krisen stellt sich die Frage nach einem angemessenen Handeln jeder*jedes Einzelnen einer- seits und nach angemessenem politischem Handeln andererseits}

Wie können Lösungsansätze im Umgang mit Krisen aussehen? Wie die Corona- und die Klimakrise zeigen, stellt sich diese Frage sowohl für jede*n Einzelne*n (was kann ich selbst tun?) als auch übergeordnet auf der politischen Ebene (welche Regelungen und Strategien werden in der Politik getroffen und verfolgt?). Jede*r Einzelne kann etwa durch das Einhalten der AHA-Regeln zur Eindämmung der Coronapandemie beitragen; auf politischer Ebene werden z. B. Entscheidungen hinsichtlich Kontaktbeschränkungen wie auch zur Umsetzung der Test- oder der Impfstrategie getroffen - die dann wieder jede*n Einzelne*n betreffen.

Klimafreundliches Handeln der*des Einzelnen kann sich z. B. in geringem Fleischkonsum, der Nutzung des öffentlichen Nahverkehrs oder dem Verzicht auf Flugreisen zeigen. Die übergeordneten klimarelevanten Entscheidungen, etwa zur jeweiligen Energie-, Verkehrs- oder Landwirtschaftspolitik, werden auf politischer Ebene getroffen. Lösungsorientierte Veränderungsprozesse müssen bei beiden Krisen also sowohl auf einer individuellen als auch auf einer systemisch-politischen Ebene ansetzen.

\section{Schutz und Anpassung als bedeutsame Lösungsansätze}

Im Umgang mit der Klimakrise werden in der Wissenschaft und in der Politik Schutzmaßnahmen und Anpassungsmaßnahmen voneinander unterschieden:

Klimaschutz (engl. mitigation) zielt darauf ab, die weltweiten Treibhausgasemissionen deutlich zu senken, da ein weiterer Temperaturanstieg auf der Erde schwerwiegende Konsequenzen für Mensch und Umwelt hätte. Beispiele für Schutzmaßnahmen in Deutschland sind die Förderung erneuerbarer Energien (u.a. Ausstieg aus der Kohleenergie), der Ausbau des öffentlichen Personennahverkehrs oder die Wiedervernässung, d. h. das Ergreifen von Maßnahmen zur Anhebung des Wasserstandes, von Mooren.

Klimaanpassung (engl. adaptation) bezeichnet bestimmte Strategien, um Ökosysteme, die Gesellschaft und die Wirtschaft widerstandsfähiger zu machen gegenüber den bereits jetzt spürbaren oder absehbaren Auswirkungen des Klimawandels. Zu diesen Auswirkungen zählen z. B. eine Zunahme von Extremwetterereignissen in vielen Teilen der Erde, der Anstieg des Meeresspiegels, in Deutschland z. B. regionale Hitzebelastung, Wasserknappheit oder steigende Hochwassergefahr. Beispiele für Anpassungsmaßnahmen in Deutschland sind z. B. Hochwasserschutz- und Waldumbauprogramme oder die Anlage von Grünflächen in Städten. 
Auch im Umgang mit der Coronakrise greift man auf Schutz- und Anpassungsmaßnahmen zurück: Schutzmaßnahmen zur Verringerung der Ausbreitung des Virus sind z. B. die „AHA-Regeln“ oder Impfungen und Teststrategien. Auf einer grundsätzlicheren Ebene kann auch der Schutz der Lebensräume von Wildtieren als Schutzmaßnahme gegenüber Pandemien angesehen werden, da so vermieden werden kann, dass Viren von Wildtieren auf den Menschen übertragen werden. Anpassungsmaßnahmen sind z. B. der Ausbau der Behandlungskapazitäten von Krankenhäusern oder die Entwicklung von Medikamenten und Impfstoffen.

Quellen:

Künzel, V. \& L. Schäfer (2020): Fünf Lehren aus der Coronakrise. Katastrophenmanagement und gesellschaftliche Widerstandsfähigkeit. In: oekom e.V. - Verein für ökologische Kommunikation (Hrsg.): Die Coronakrise. Stimulanz für die Große Transformation? oekom, München. S. 63-68.

Lenton, T., H. Held, E. Kriegler, J. Hall, W. Lucht, S. Rahmstorf \& H. J. Schellnhuber (2008): Tipping elements in the Earth's climate system. In: Proceedings of the National Academy of Sciences 105(6). S. 1786-1793. DOI: https://doi. org/10.1073/pnas.0705414105

PIK (Potsdam-Institut für Klimafolgenforschung) (2019): Kipppunkte im Klimasystem. Eine kurze Übersicht. Potsdam. http:// www.pik-potsdam.de/-stefan/Publications/Kipppunkte\%20im\%20Klimasystem\%20-\%20Update\%202019.pdf (07.06.2021)

\section{M4 | Kernthesen der Teilnehmer*innen der Podiumsdiskussion}

Prof. Dr. Elke Hertig, Professorin für regionalen Klimawandel und Gesundheit (Universität Augsburg)

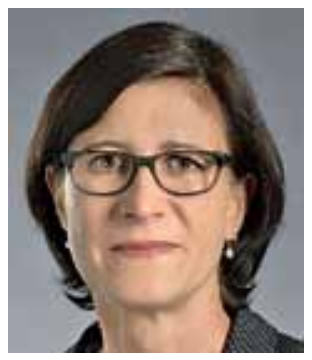

Kurzporträt: Nach ihrem Geographiestudium wurde Elke Hertig (EH) im Fach Klimatologie an der Universität Würzburg promoviert, anschließend habilitierte sie am Geographischen Institut der Universität Augsburg. ${ }^{4}$ Seit 2019 ist sie Professorin für Regionalen Klimawandel und Gesundheit an der Medizinischen Fakultät der Universität Augsburg. Sie beschäftigt sich u. a. mit den gesundheitlichen Auswirkungen von Wetter, Luftschadstoffen und Klima unter Fortgang des Klimawandels.

These „EH 1“(,EH“steht für „Elke Hertig“-auch diefolgenden Kürzel sind immer die Initialen der Expert*innen): „Nach Bewältigung der Pandemie werden die Menschen wieder in ihre alten Verhaltensmuster zurückkehren und genauso konsumieren, Urlaubsreisen unternehmen, etc. Die Bewältigung der Klimakrise bleibt reine Fiktion, wenn nicht maßgebliche Transformationsprozesse in Gang kommen."

\section{These „EH 2“}

„Die menschliche Gesundheit kann nur geschützt werden, wenn wir die Natur schützen. Ohne eine gesamtheitliche Betrachtung werden Klimawandel und Pandemien uns immer stärker treffen."

Thomas LOSTER, Diplom-Geograph (Hochschule für angewandte Wissenschaften München)

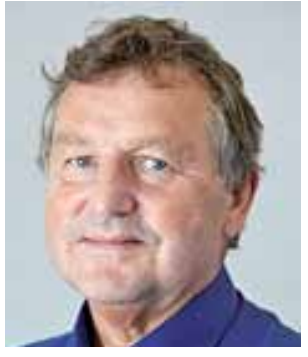

Kurzporträt: Der Diplom-Geograph Thomas Loster (TL) ist Experte für Klimawandel, Naturgefahren, Entwicklungszusammenarbeit und nachhaltige Entwicklung. Er vertrat die Rückversicherung Munich Re und deren Stiftung bei über 20 Weltklimagipfeln. Als Fachmann für Entwicklungszusammenarbeit lehrt er an der Hochschule für angewandte Wissenschaften in München. Sein Thema dort: Nachhaltige Lösungen für globale Herausforderungen.

\section{These ,TL 1“:}

„Wenn wir uns streng an das Pariser Abkommen halten, verschwenden wir wertvolle Zeit und gefährden unser Klima nachweislich. Wir brauchen starkes, rasches Handeln, top-down und gut verschränkt mit bottom-up. ${ }^{5}$ Sonst kriegen wir das Wunschklima nicht hin."

\footnotetext{
4 „Promovieren“ bedeutet einen Doktortitel zu erwerben; die „Habilitation“ ist eine an die Promotion anschließende weitere wissenschaftliche Qualifikation auf dem Weg zur Professur.

5 Im Kontext der Diskussionen um die Klimakrise bezeichnen top-down-Ansätze klimapolitische Bestrebungen von oben, also bspw. auf internationaler Maßstabsebene konkrete Verpflichtungen für einzelne Staaten abzuleiten (z. B. das 1,5 $\left.{ }^{\circ} \mathrm{C}-Z i e l\right)$. Bottom-up-Ansätze hingegen beschreiben Innovations- sowie Veränderungsprozesse von unten, also bspw. auf lokaler oder regionaler Maßstabsebene, die klimaschützende oder -anpassende Wirkung entfalten. Hierunter fallen bspw. klimaneutrale Verfahren oder Produkte.
} 
These ,TL 2":

„Es ist hochriskant, wenn Länder in Zeiten von Corona extreme Versprechen für $\mathrm{CO}_{2}$-Reduktionen machen, für die es noch nicht einmal Prozesse und realistische Pfade gibt."

\title{
Luisa Neubauer, Klimaaktivistin (Fridays for Future)
}

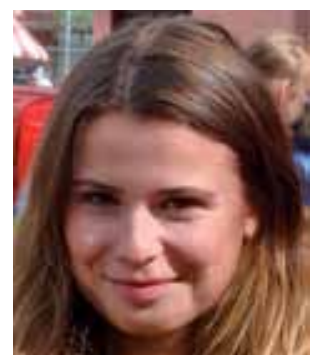

Kurzporträt: Luisa Neubauer ist Klimaaktivistin und eine der Hauptorganisator*innen der Fridays for Future. Sie setzt sich für die Ziele der Klimapolitik ein, die im Übereinkommen von Paris von 2015 formuliert sind, und vertritt wachstumskritische Positionen. 2020 schloss sie ihr Studium des Faches Geographie an der Universität Göttingen mit dem Bachelor of Science ab.

\begin{abstract}
These „LN 1“
„Zu Beginn der Pandemie sagte man mit Blick auf das Klima, dass \#Corona beweisen würde, dass man Krisen bewältigen kann, wenn man will. Jetzt stellen wir fest, dass Bewältigen-Wollen nicht ausreicht. Man muss es auch können. Das kann mit der \#Klimakrise noch richtig heiter werden." (https://twitter.com/luisamneubauer/status/1380468274597658627;09.04.2021)
\end{abstract}

\section{These "LN 2“}

„Wir erleben einen ,Jetzismus', also eine Gegenwartsversessenheit in neuer Qualität. Das ist nicht wirklich hilfreich, wenn es darum geht sich vor zukünftigen Krisen zu schützen." (https://rp-online.de/politik/deutschland/luisa-neubauer-interview-wir-koennen-nicht-gesund-bleiben-in-einer-kranken-umwelt aid-56416237; 24.02.2021)

Prof. Dr. Patrick SAKDaPOlRaK, Professor für Bevölkerungsgeographie und Demographie (Uni Wien)

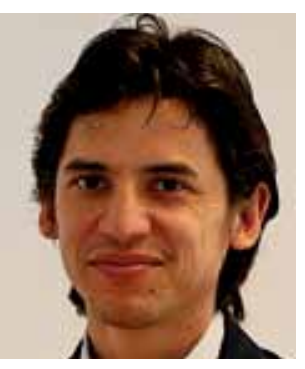

Kurzporträt: Patrick Sakdapolrak hat in Heidelberg Geographie studiert und in Bonn promoviert. Seit 2016 ist er Professor für Bevölkerungsgeographie und Demographie an der Universität Wien. Er beschäftigt sich mit dem Umgang mit Risiken von verwundbaren Gruppen im Globalen Süden. Ein besonderer Fokus seiner Forschung liegt auf dem Thema Migration im Kontext des globalen Umweltwandels.

\section{These „PS 1“}

„Trotz des politischen Versprechens den Post-Pandemie Wiederaufbau grün und nachhaltig zu gestalten, ist die Rückkehr zum business as usual ein wahrscheinliches Szenario. Damit ist auch die Erreichung der nachhaltigen Entwicklungsziele (SDGs) ernsthaft in Gefahr.“

\section{These „PS 2“}

„Die Corona-Pandemie wird die Finanzierungslücke für die notwendigen Anpassungsanstrengungen gegen über den Folgen des Klimawandels vergrößern. Bei gleichzeitiger Corona-bedingter Schwächung der Anpassungsmöglichkeiten der Bevölkerung, wird sich die Ungleichheit in der Betroffenheit gegenüber Klimafolgen global weiter verstärken."

M5 | Meinungsstrahl: Welcher These der Expert*innen stimmst du vor der Podiumsdiskussion wie stark zu?

Schreibe die Kürzel für die einzelnen Thesen an die passende Stelle auf dem Strahl.

ich stimme sehr stark zu ich stimme

gar nicht zu 


\section{Arbeitsblatt 2: Podiumsdiskussion und Reflexion}

\section{Aufgaben:}

1. Schaut euch die Podiumsdiskussion an.

Sofern ihr an der Liveübertragungteilnehmen könnt, bringt euch mit euren vorbereiteten Fragen und Kommentaren in die Diskussion ein (voraussichtlich können nicht alle Wortbeiträge auch tatsächlich vom Moderator aufgegriffen werden) und notiert stichwortartig dazu passende Aussagen der Diskussionsteilnehmer*innen. Falls ihr die Aufzeichnung der Podiumsdiskussion anschaut, notiert stichwortartig Aussagen der Diskussionsteilnehmer*innen, die hinsichtlich eurer vorbereiteten Fragen und Kommentare wichtig sind.

2. Notiert mindestens drei Argumente aus der Diskussion, die euch besonders überzeugt haben, und mindestens drei, die euch weniger überzeugt haben.

3. Ordnet die Kernthesen aus M4 nach der Podiumsdiskussion erneut auf einem Meinungsstrahl (M6) an. Erläutert in Stichworten unter Bezug auf die Inhalte der Podiumsdiskussion, ob und warum sich Veränderungen bei euren Zuordnungen ergeben haben.

\section{M6 | Meinungsstrahl: Welcher These der Expert*innen stimmst du nach der Podiumsdiskussion wie stark zu?}

Schreibe die Kürzel für die einzelnen Thesen an die passende Stelle auf dem Strahl.

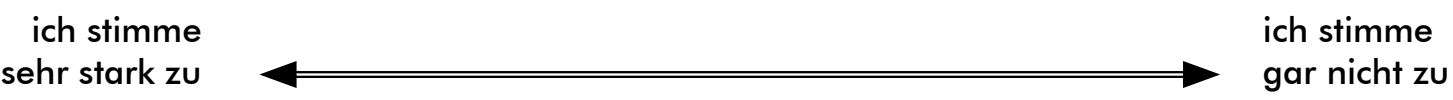

\section{Material für die weiterführende Diskussion}

\section{M7 | Zusammenhänge multipler Krisen}

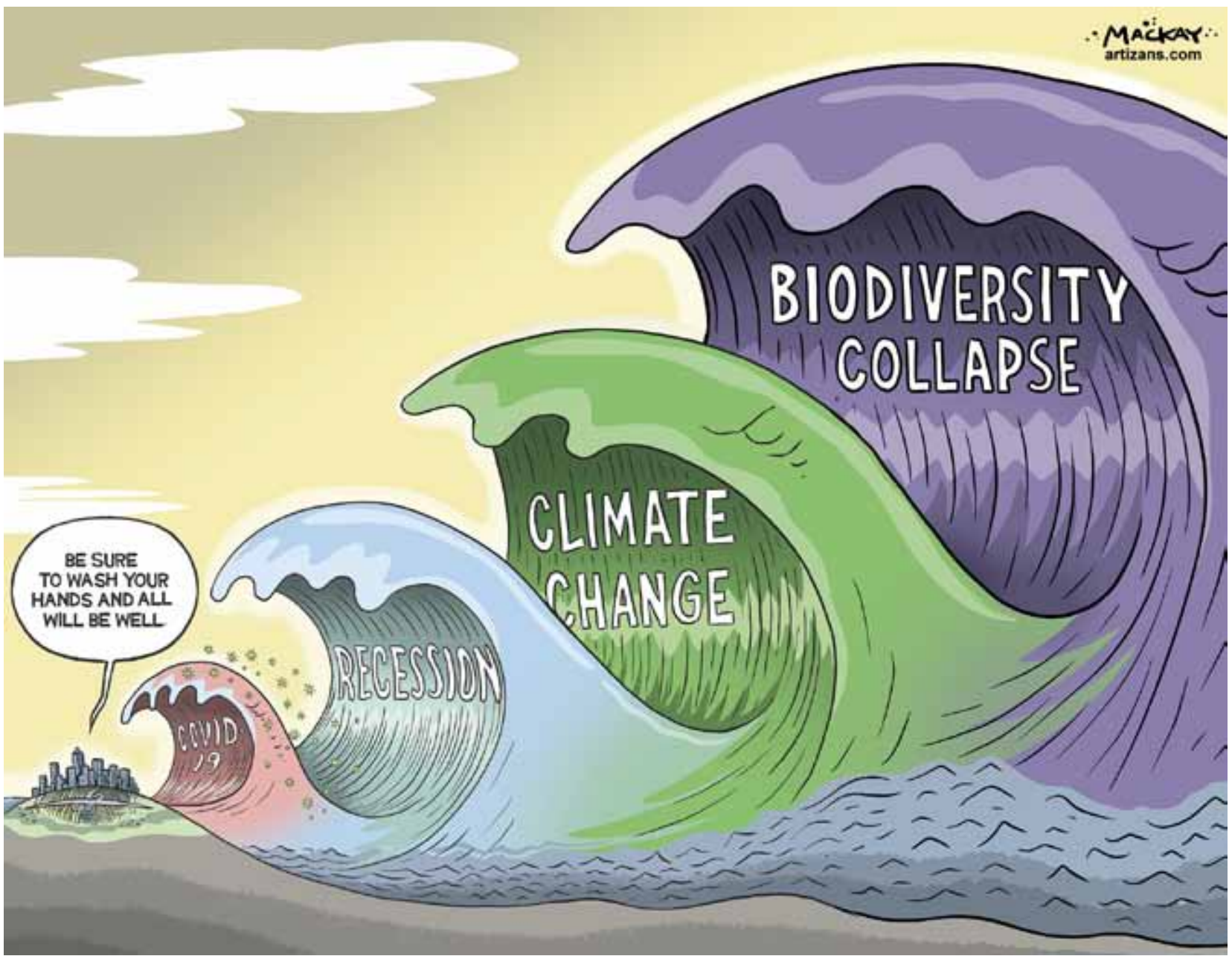

